\title{
Estimating maturity of paddy using RGB colour space
}

\begin{abstract}
Colour plays important role in evaluating quality and maturity level of many agricultural products. Immature paddy has been defined as kernels or pieces of grain kernels that are not fully developed and are yellow-green in colour. Immature paddy will caused broken rice and losses during storage. This paper presents a new technique of paddy maturity estimation using various colour indices extracted from RGB colour spaces. Paddy samples were taken during 96 and 98 Days After Planting (DAP). Results has shown that there are significant relationships between all of the colour indices with the DAP. The strongest significant relationship can be gathered from G-B colour index, with $\mathrm{R}=-0.924$ ( $\mathrm{p}>0.001)$. A linear regression model was later being developed based on G-B colour index defined as MATURITY=-16.95 (G-B)+101.61.
\end{abstract}

Keyword: Paddy; Maturity; RGB; Colour indices; Image analysis 